\title{
SCIENTIFIC REPRTS OPEN A diagnostic tool for malaria based
on computer software
}

Received: 28 May 2015

Accepted: 19 October 2015

Published: 12 November 2015

\author{
Manas Kotepui ${ }^{1}$, Kwuntida Uthaisar ${ }^{1}$, Bhukdee Phunphuech ${ }^{2}$ \& Nuoil Phiwklam²
}

Nowadays, the gold standard method for malaria diagnosis is a staining of thick and thin blood film examined by expert laboratorists. It requires well-trained laboratorists, which is a time consuming task, and is un-automated protocol. For this study, Maladiag Software was developed to predict malaria infection in suspected malaria patients. The demographic data of patients, examination for malaria parasites, and complete blood count (CBC) profiles were analyzed. Binary logistic regression was used to create the equation for the malaria diagnosis. The diagnostic parameters of the equation were tested on 4,985 samples (703 infected and 4,282 control samples). The equation indicated $81.2 \%$ sensitivity and $80.3 \%$ specificity for predicting infection of malaria. The positive likelihood and negative likelihood ratio were $4.12(95 \% \mathrm{Cl}=4.01-4.23)$ and $0.23(95 \% \mathrm{Cl}=0.22-0.25)$, respectively. This parameter also had odds ratios ( $\mathrm{P}$ value $<0.0001, \mathrm{OR}=17.6,95 \% \mathrm{Cl}=16.0-19.3$ ). The equation can predict malaria infection after adjust for age, gender, nationality, monocyte (\%), platelet count, neutrophil (\%), lymphocyte (\%), and the RBC count of patients. The diagnostic accuracy was 0.877 (Area under curve, AUC) $(95 \% \mathrm{Cl}=0.871-0.883)$. The system, when used in combination with other clinical and microscopy methods, might improve malaria diagnoses and enhance prompt treatment.

Malaria is a potential medical emergency and should be treated immediately because delays in diagnosis and treatment are leading causes of death in many countries ${ }^{1}$. The first protocol to the diagnosis of malaria infection is a clinical diagnosis based on signs and symptoms of malaria patients which were most widely practiced. The signs and symptoms of malaria include fever, headache, weakness, myalgia, chills, dizziness, abdominal pain, diarrhea, anorexia, and pruritus ${ }^{2}$. However, a clinical diagnosis of malaria from symptoms of malaria patients is still inaccurate because of the non-specific symptoms similar to viral or bacterial infections, and other febrile illnesses which may impair diagnostic specificity ${ }^{3,4}$.

To gain a more precise diagnosis, malaria is diagnosed using microscopic diagnosis by the gold standard method which is the staining of thin and thick peripheral blood smears ${ }^{5}$, and other techniques such as the quantitative buffy coat (QBC) method ${ }^{6}$. The rapid diagnostic test (RDT) is another method that detects malaria antigens in a small amount of blood by immunochromatographic assay impregnated on a test strip ${ }^{7}$. The RDT dipstick was a commercially available and frequently uses routine diagnostic tool such as OptiMAL ${ }^{8,9}$ ICT $^{10}$, Para-HIT- $\mathrm{f}^{11}$, ParaScreen ${ }^{12}$, SD Bioline ${ }^{13}$, Paracheck ${ }^{14}$. Molecular diagnostic methods, such as polymerase chain reaction (PCR) was also used with higher sensitivity when compared to the blood film examination ${ }^{15}$. Some advantages and disadvantages of these methods related to accuracy, precision, sensitivity, specificity, time consumed, cost-effectiveness, and the need for skilled microscopists have been described.

Changes in hematological parameters are well-known features of malaria infection. These changes involve various cell types such as RBCs, leucocytes and thrombocytes ${ }^{16,17}$. Malaria infected patients tend to have significantly lower RBC counts, platelets count, leukocyte, lymphocytes count, and Hb level, while monocyte and neutrophil counts were higher in non-malaria infected patients ${ }^{16-19}$. Nowadays, the gold standard method for malaria detection is examined by expert laboratorists. It requires well-trained labolatorists, it is time consuming, and is an unautomated protocol. In routine laboratory tests, a complete

${ }^{1}$ Medical Technology Program, School of Allied Health Sciences and Public Health, Walailak University, Nakhon Si Thammarat, 80161, Thailand. ${ }^{2}$ Medical Technology Laboratory, Phop Phra Hospital, Phop Phra District, Tak Province, 63160. Thailand. Correspondence and requests for materials should be addressed to M.K. (email: manas. ko@wu.ac.th) 


\begin{tabular}{|l|c|c|c|c|c|c|}
\hline Parameters & B & S.E. & Wald & df & Sig. & Exp(B) \\
\hline Constant & 2.936 & 0.034 & 7377.426 & 1 & 0.000 & 18.848 \\
\hline Gender & -0.533 & 0.088 & 36.503 & 1 & 0.000 & 0.587 \\
\hline Age & 0.013 & 0.003 & 26.858 & 1 & 0.000 & 1.013 \\
\hline Nationality & 0.814 & 0.087 & 86.810 & 1 & 0.000 & 2.256 \\
\hline WBC & 0.032 & 0.014 & 5.564 & 1 & 0.018 & 1.033 \\
\hline Neutrophil (\%) & -0.022 & 0.006 & 12.408 & 1 & 0.000 & 0.979 \\
\hline Lymphocyte (\%) & 0.036 & 0.006 & 38.186 & 1 & 0.000 & 1.037 \\
\hline Platelet & 0.023 & 0.001 & 916.920 & 1 & 0.000 & 1.023 \\
\hline Monocyte (\%) & -0.093 & 0.013 & 52.435 & 1 & 0.000 & 0.911 \\
\hline Eosinophil (\%) & -0.034 & 0.016 & 4.692 & 1 & 0.030 & 0.967 \\
\hline Basophil (\%) & -0.044 & 0.055 & 0.654 & 1 & 0.419 & 0.957 \\
\hline RBC & 0.930 & 0.185 & 25.381 & 1 & 0.000 & 2.536 \\
\hline Hb & -0.009 & 0.065 & 0.017 & 1 & 0.895 & 0.991 \\
\hline MCV & 0.020 & 0.014 & 1.996 & 1 & 0.158 & 1.020 \\
\hline MCH & 0.101 & 0.045 & 5.126 & 1 & 0.024 & 1.106 \\
\hline MCHC & 0.063 & 0.028 & 5.024 & 1 & 0.025 & 1.065 \\
\hline RDW & 11.948 & 1.501 & 63.387 & 1 & 0.000 & 0.000 \\
\hline Constant & & 0.026 & 6.043 & 1 & 0.014 & 1.067 \\
\hline & & & 1 & 1 & 1 \\
\hline
\end{tabular}

Table 1. Logistic regression of demographic and complete blood count variables with the status of malaria infection.

blood count $(\mathrm{CBC})$ is almost always requested without exception as part of the routine investigation in febrile patients. In previously published research, there were some reports using routine automated hematology analyzers for presumptive diagnosis of malaria infection. Most studies are those regarding abnormal depolarizing patterns of the Cell-Dyn hematology analyzer (Abbott Diagnostics, Santa Clara, $\mathrm{CA})^{20-24}$. Therefore, this study aimed to develop diagnosis software assisting in the diagnosis of malaria infection in suspected-malaria patients. The software needs to be easy-to-use, include batch screening for routine diagnosis, and have high sensitivity and specificity.

\section{Results}

Patient characteristics. Between January $1^{\text {st }} 2008$ and December $1^{\text {st }} 2012$, data from of 43,899 cases of patients suspected with malaria infection was analyzed. Of these cases, $3,082(7 \%)$ were patients with malaria infection, whereas 40,817 (93\%) were patients with non-malaria infection according to thick and thin film examinations.

Regression model. The regression model was performed to create the equation for malaria diagnosis. In the first, all patients' parameters previously collected were entered and calculated (Table 1). Variables from the first step that showed significant association with the status of malaria infection ( $\mathrm{p}$ value $<0.05$ ) such as gender, age, nationality, neutrophil (\%), lymphocyte (\%), platelet count, monocyte (\%), and the $\mathrm{RBC}$ count were then entered into the second step of the logistic regression analysis (Table 2). After the second regression analysis, the equation for malaria diagnosis was as shown below:

$\mathrm{z}=(-7.499)-0.373$ (gender) $+0.018($ age $)+1.019$ (nationality) +0.017 (neutrophil) +0.017 (lymphocyte $)+0.022$ (platelet $)-0.033($ monocyte $)+0.691($ RBC $)$

Prob (non-malaria infection $)=1 /\left(1+\left(2.178^{(-z)}\right)\right)$

Prob $($ malaria infection $)=1-$ Prob (non-malaria infection)

$\mathrm{Z}=$ constant of regression analysis

After ROC analysis was performed (Fig. 1), the suitable sensitivity and specificity of the equation to detect malaria infections was B value $<0.2208453$. The equation had high sensitivity $(81.2 \%)$ and specificity $(80.3 \%)$ to detect infection of malaria parasites (Table 3 ). The positive likelihood ratio was 4.12 $(95 \% \mathrm{CI}=4.01-4.23)$, while the negative likelihood ratio was $0.23(95 \% \mathrm{CI}=0.22-0.25)$. This parameter also had good odd ratios ( $\mathrm{P}$ value $<0.0001, \mathrm{OR}=17.6, \mathrm{CI}=16.0-19.3$ ). It indicates that the equation can predict malaria infection after adjust for age, gender, nationality, neutrophil (\%), lymphocyte (\%), monocyte (\%), platelet count, and RBC count of patients. The diagnostic accuracy was 0.877 (area under curve; $\mathrm{AUC}=0.877,95 \% \mathrm{CI}=0.871-0.883$ ). 


\begin{tabular}{|l|c|c|c|c|c|c|}
\hline & B & S.E. & Wald & df & Sig. & $\operatorname{Exp(B)}$ \\
\hline Constant & 2.585 & 0.019 & 18949.324 & 1 & 0.000 & 13.266 \\
\hline Gender & -0.373 & 0.048 & 61.381 & 1 & 0.000 & 0.689 \\
\hline Age & 0.018 & 0.001 & 174.893 & 1 & 0.000 & 1.018 \\
\hline Nationality & 1.019 & 0.048 & 457.826 & 1 & 0.000 & 2.770 \\
\hline Neutrophil (\%) & 0.017 & 0.004 & 19.344 & 1 & 0.000 & 1.017 \\
\hline Lymphocyte (\%) & 0.057 & 0.004 & 206.379 & 1 & 0.000 & 1.059 \\
\hline Platelet & 0.022 & 0.000 & 3502.501 & 1 & 0.000 & 1.023 \\
\hline Monocyte (\%) & -0.033 & 0.007 & 24.421 & 1 & 0.000 & 0.968 \\
\hline RBC & 0.691 & 0.031 & 488.879 & 1 & 0.000 & 1.996 \\
\hline Constant & -7.499 & 0.417 & 322.765 & 1 & 0.000 & 0.001 \\
\hline
\end{tabular}

Table 2. Logistic regression of significant variables with the status of malaria infection.

\section{ROC Curve}

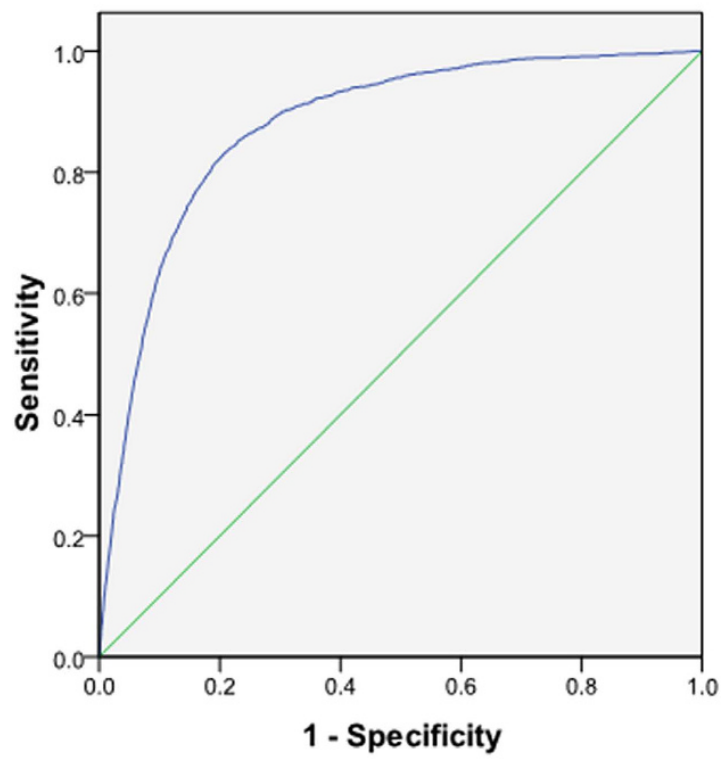

Diagonal segments are produced by ties.

Figure 1. The ROC curve showed the sensitivity and specificity of the equation to detect malaria infection.

\begin{tabular}{|l|c|}
\hline Performance of test & Value \\
\hline Sensitivity (95\% CI) & $81.2(79.8-82.6)$ \\
\hline Specificity (95\% CI) & $80.3(79.9-80.7)$ \\
\hline PPV (95\% CI) & $23.7(22.9-24.6)$ \\
\hline NPV (95\% CI) & $98.3(98.1-98.4)$ \\
\hline Positive Likelihood Ratio (95\% CI) & $4.12(4.01-4.23)$ \\
\hline Negative Likelihood Ratio (95\% CI) & $0.23(0.22-0.25)$ \\
\hline Diagnostic accuracy (95\% CI) & $87.7(87.1-88.3)$ \\
\hline
\end{tabular}

Table 3. Sensitivity, specificity, PPV, NPV, and diagnostic accuracy of the Maladiag software. 


\section{Discussion}

In the first regression step, the parameter that was significant in the equation included gender, age, nationality, neutrophil (\%), lymphocyte (\%), platelet count, monocyte (\%), and RBC. This is due to these parameters incurring significant changes during malaria infection that can result differently from non-infected malaria patients. The previous study of patients in Thailand showed that gender, age, nationality, neutrophil (\%), lymphocyte (\%), platelet count, monocyte (\%), and RBC significantly changed from malaria infections. A previous study showed patients with higher a WBC count compared with community controls ${ }^{10}$. The most common complication during malaria infection is thrombocytopenia ${ }^{6,11-13}$

An automated blood cell counter (ACC) is a practical tool for malaria diagnosis ${ }^{21}$. In this study, the sensitivity, specificity, likelihood ratio and diagnostic accuracy for all the hematological parameters were determined. The software had high sensitivity and specificity $(81.3,80.1 \%$, respectively) which was higher than the previous study that used a Cell-Dyn ${ }^{\circledR} 3500$, and analyzed depolarized laser light (DLL) to detect malaria infection, with an overall sensitivity of $72 \%$ and specificity of $96 \%{ }^{22}$. However, the sensitivity and specificity were lower than the previous study that used a Cell-Dyn ${ }^{\circledR} 3500$ apparatus to detect malaria pigment (hemozoin) in monocytes, and showed a sensitivity of $95 \%$ and specificity of $88 \%$, compared with the gold-standard blood smear ${ }^{20}$. the sensitivity and specificity were lower than the previous study that used a Beckman Coulter ACC to detect increases in activated monocytes by volume, conductivity, and scatter (VCS), with $98 \%$ sensitivity and $94 \%$ specificity ${ }^{25}$.

Similar to the RDT, this software will reduce the time of malaria diagnosis, with the result obtainable in $5-20 \mathrm{~min}^{7}$. In addition, this software can be further used to diagnose malaria in automated instruments. The sensitivity and specificity of this software when compared to RDTs were lower than ParaSight $\mathrm{F}^{26-28}$, which yields sensitivity from 77 to $98 \%$ and specificity from 83 to $98 \%$ for P. falciparum. It was also lower when compared to the sensitivity and specificity of the Opti-MAL test ${ }^{29}$ which yields sensitivity of $94 \%$ for P. falciparum and specificity of $100 \%$ for P. falciparum. Although the efficiency of this software was lower in terms of sensitivity and specificity than those methods currently used in routine laboratories, it served as another way to help diagnose suspected malaria patients. Further studies are required to improve the sensitivity, specificity, and validate this software. In addition, the software, when used in combination with other clinical and microscopy methods, might help to improve malaria diagnoses in endemic areas.

\section{Methods}

Ethical approval. This study protocol was approved by The Ethical Clearance Committee on Human Rights Related to Research Involving Human Subjects of Walailak University. The informed consent was not obtained from all subjects but the name and Hospital Number (HN) of patients were not revealed.

Data collection. The data used in this study were collected from the Medical Technology Laboratory Unit, Phobphra Hospital, Tak Province. The methods were carried out in accordance with the approved guidelines. The medical record keeping system in the Department at The Phobphra Hospital is electronic. The data of all patients diagnosed with malaria between January $1^{\text {st }} 2008$ and December $1^{\text {st }} 2012$ were extracted and enter into the SPSS program. Demographic, clinical and laboratory data of all the patients were also collected. Leukocyte, red blood cells, and platelet counts were measured using an automatic cell counter. Other laboratory examinations including RBC, $\mathrm{Hb}, \mathrm{MCV}, \mathrm{MCH}, \mathrm{MCHC}, \mathrm{RDW}$, and platelet counts were also collected. The standard procedure used for the diagnosis of malaria is the examination of thick and thin blood smears for malaria parasites by the Wright and Giemsa staining under light microscopy by laboratorists at 1,000 magnification.

Statistical methods. The Kolmogorov-Smirnov test was performed to test the normal distribution of the data. Binary logistic regression was performed to find out which parameter was suitable for predicting malaria infections; the logistic regression model was performed using the leukocyte count, RBC count, $\mathrm{Hb}, \mathrm{MCV}, \mathrm{MCH}, \mathrm{MCHC}$, $\mathrm{RDW}$, and platelet counts as covariates and using the status of malaria infection (found or not found) as a dependent variable. Data analysis was performed using SPSS ver. 11.5 (SPSS Inc., Chicago, IL, USA). Any parameters that were statistically significant will be put into the second step of regression analysis. Diagnostic accuracy of hematological parameters was measured by a receiver operating characteristic (ROC) and then calculating the sensitivity, specificity, positive/negative predictive values and odds ratios with $95 \%$ confidence intervals.

\section{References}

1. World Health Organization, Malaria., (2015) Available at: http://www.who.int/mediacentre/factsheets/fs094/en/. (Accessed: $2^{\text {th }}$ January 2015).

2. Looareesuwan, S. Malaria. In: Looareesuwan S, W. P. Clinical Tropical Medicine. 1st ed. edn, 5-10 (Medical Media, 1999).

3. Mwangi, T. W., Mohammed, M., Dayo, H., Snow, R. W. \& Marsh, K. Clinical algorithms for malaria diagnosis lack utility among people of different age groups. Trop Med Int Health 10, 530-536 (2005)

4. Reyburn, H. et al. Overdiagnosis of malaria in patients with severe febrile illness in Tanzania: a prospective study. BMJ 329, 1212 (2004).

5. Ngasala, B. et al. Impact of training in clinical and microscopy diagnosis of childhood malaria on anti-malarial drug prescription and health outcome at primary health care level in Tanzania: a randomized controlled trial. Malar J 7, 199 (2008). 
6. Bhandari, P. L., Raghuveer, C. V., Rajeev, A. \& Bhandari, P. D. Comparative study of peripheral blood smear, quantitative buffy coat and modified centrifuged blood smear in malaria diagnosis. Indian J Pathol Microbiol 51, 108-112 (2008).

7. Wongsrichanalai, C., Barcus, M. J., Muth, S., Sutamihardja, A. \& Wernsdorfer, W. H. A review of malaria diagnostic tools: microscopy and rapid diagnostic test (RDT). The American journal of tropical medicine and hygiene 77, 119-127 (2007).

8. Tagbor, H., Bruce, J., Browne, E., Greenwood, B. \& Chandramohan, D. Performance of the OptiMAL dipstick in the diagnosis of malaria infection in pregnancy. Ther Clin Risk Manag 4, 631-636 (2008).

9. Zerpa, N. et al. Evaluation of the OptiMAL test for diagnosis of malaria in Venezuela. Invest Clin 49, 93-101 (2008).

10. Ratsimbasoa, A. et al. Evaluation of two new immunochromatographic assays for diagnosis of malaria. Am J Trop Med Hyg 79, 670-672 (2008).

11. McMorrow, M. L., Masanja, M. I., Abdulla, S. M., Kahigwa, E. \& Kachur, S. P. Challenges in routine implementation and quality control of rapid diagnostic tests for malaria-Rufiji District, Tanzania. Am J Trop Med Hyg 79, 385-390 (2008).

12. Endeshaw, T. et al. Evaluation of light microscopy and rapid diagnostic test for the detection of malaria under operational field conditions: a household survey in Ethiopia. Malar J 7, 118 (2008).

13. Lee, S. W., Jeon, K., Jeon, B. R. \& Park, I. Rapid diagnosis of vivax malaria by the SD Bioline Malaria Antigen test when thrombocytopenia is present. J Clin Microbiol 46, 939-942 (2008).

14. Harvey, S. A. et al. Improving community health worker use of malaria rapid diagnostic tests in Zambia: package instructions, job aid and job aid-plus-training. Malar J 7, 160 (2008).

15. Vo, T. K. et al. Evaluation of a real-time PCR assay for malaria diagnosis in patients from Vietnam and in returned travelers. Trans R Soc Trop Med 101, 422-428 (2007).

16. Bakhubaira, S. Hematological parameters in severe complicated Plasmodium falciparum malaria among adults in Aden. Turk $J$ Haematol 30, 394-399 (2013).

17. Maina, R. N. et al. Impact of Plasmodium falciparum infection on haematological parameters in children living in Western Kenya. Malar J 9, S4 (2010).

18. van Wolfswinkel, M. E. et al. Predictive value of lymphocytopenia and the neutrophil-lymphocyte count ratio for severe imported malaria. Malar J 12, 101 (2013).

19. Erhart, L. M. et al. Hematologic and clinical indices of malaria in a semi-immune population of western Thailand. Am J Trop Med Hyg 70, 8-14 (2004).

20. Hanscheid, T., Melo-Cristino, J. \& Pinto, B. G. Automated detection of malaria pigment in white blood cells for the diagnosis of malaria in Portugal. Am J Trop Med Hyg 64, 290-292 (2001).

21. de Langen, A. J. et al. Automated detection of malaria pigment: feasibility for malaria diagnosing in an area with seasonal malaria in northern Namibia. Trop Med Int Health 11, 809-816 (2006).

22. Mendelow, B. V. et al. Automated malaria detection by depolarization of laser light. Br J Haematol 104, 499-503 (1999).

23. Hänscheid, T., Pinto, B. G., Pereira, I., Cristino, J. M. \& Valadas, E. Avoiding misdiagnosis of malaria: a novel automated method allows specific diagnosis, even in the absence of clinical suspicion. Emerg Infect Dis 5, 836-838 (1999).

24. Scott, C. S. et al. Automated detection of malaria-associated intraleucocytic haemozoin by Cell-Dyn CD4000 depolarization analysis. Clin Lab Haematol 25, 77-86 (2003).

25. Briggs, C., Da Costa, A., Freeman, Lyn., Aucamp, I., Ngubeni, B. \& Machin, S. J. Development of an automated malaria discriminant factor using VCS technology. Am J Clin Pathol 126, 691-698 (2006).

26. Beadle, C. et al. Diagnosis of malaria by detection of Plasmodium falciparum HRP-2 antigen with a rapid dipstick antigencapture assay. Lancet 343, 564-568 (1994).

27. Kodisinghe, H. M. et al. The ParaSight-F dipstick test as a routine diagnostic tool for malaria in Sri Lanka. Trans R Soc Trop Med Hyg 91, 398-402 (1997).

28. Laban, N. M. et al. Comparison of a PfHRP2-based rapid diagnostic test and PCR for malaria in a low prevalence setting in rural southern Zambia: implications for elimination. Malaria journal 14, 25, doi: 10.1186/s12936-015-0544-3 (2015).

29. Palmer, C. J. et al. Evaluation of the OptiMAL test for rapid diagnosis of Plasmodium vivax and Plasmodium falciparum malaria. Journal of clinical microbiology 36, 203-206 (1998).

\section{Acknowledgements}

This research was funded by grants from Walailak University (Predictive Risk Factors for Diseases and Health Impact Research Group, WU58525). The authors were grateful and would like to specifically thank the Medical Technology Laboratory Unit, Phop Phra Hospital, for their data, which was important to this research article. The authors also thank Mrs. Premrudee Noonsang, who spent considerable time helping to develop the software.

\section{Author Contributions}

M.K. conceived the study and designed the experiments, performed the experiments, analyzed the data and wrote the report. K.U. designed the experiments and wrote the report. P.P. designed and performed the experiments. N.P. collected the data.

\section{Additional Information}

Competing financial interests: The authors declare no competing financial interests.

How to cite this article: Kotepui, M. et al. A diagnostic tool for malaria based on computer software. Sci. Rep. 5, 16656; doi: 10.1038/srep16656 (2015).

(c) (i) This work is licensed under a Creative Commons Attribution 4.0 International License. The images or other third party material in this article are included in the article's Creative Commons license, unless indicated otherwise in the credit line; if the material is not included under the Creative Commons license, users will need to obtain permission from the license holder to reproduce the material. To view a copy of this license, visit http://creativecommons.org/licenses/by/4.0/ 